\title{
Ceftazidime/Avibactam versus Polymyxin B in the Challenge of Carbapenem-Resistant Pseudomonas aeruginosa Infection
}

\author{
Juan Chen*, Qiqiang Liang*, Xinyi Chen, Jing Wu, Yanchao Wu, Gaoqin Teng (D, Man Huang (D) \\ Department of General Intensive Care Unit, The Second Affiliated Hospital of Zhejiang University School of Medicine, Hangzhou, Zhejiang, People's \\ Republic of China \\ *These authors contributed equally to this work \\ Correspondence: Man Huang, Department of General Intensive Care Unit, The Second Affiliated Hospital of Zhejiang University School of Medicine, \\ Hangzhou, Zhejiang, People's Republic of China, Tel/Fax +86 57I 897I3427, Email huangman@zju.edu.cn
}

\begin{abstract}
Purpose: Ceftazidime/avibactam (CAZ/AVI) monotherapy and polymyxin B-based combination therapy are currently two treatment options for patients with carbapenem-resistant Pseudomonas aeruginosa (CRPA) infection; however, few studies have contrasted the relative efficacy of the two antibiotic regimens. The purpose of this study was to compare the effectiveness of CAZ/AVI and polymyxin B against CRPA infection and analyze the independent predictors of 30-day mortality or survival.

Patients and Methods: This single-center retrospective observational study included patients with CRPA infection treated with CAZ/AVI or polymyxin B between January 2018 and December 2020. The primary outcomes were the 14-day and 30-day mortality. The secondary outcomes were in-hospital mortality and bacterial clearance. Baseline characteristics and outcomes were compared between the two groups, and COX regression analysis was used to identify predictors of 30-day mortality.

Results: A total of 136 patients with CRPA infection were enrolled, including 51 patients in the CAZ/AVI group and 85 patients in the polymyxin B group. The 14-day mortality $(5.9 \%$ vs $27.1 \%$, p=0.002), 30-day mortality $(13.7 \%$ vs $47.1 \%$, p $<0.001)$ and in-hospital mortality $(29.4 \%$ vs $60.0 \%, \mathrm{p}=0.001)$ in the CAZ/AVI group were significantly lower than the polymyxin B group. The bacterial clearance rate $(45.1 \%$ vs $12.9 \%, \mathrm{p}<0.001)$ in the CAZ/AVI group were higher than in the polymyxin B group. After adjustment by propensity score matching, the CAV/AVI group still had lower 30-day mortality $(14.3 \%$ vs $42.9 \%$, p=0.018) and higher bacterial clearance rate $(42.9 \%$ vs $14.3 \%, \mathrm{p}=0.018)$ than the polymyxin B group. The multivariate COX analysis showed that the age was identified as independent predictor of 30-day mortality while CAZ/AVI therapy and central venous catheterization emerged as independent predictors of 30-day survival.
\end{abstract}

Conclusion: CAZ/AVI therapy was superior to polymyxin B therapy for patients with CRPA infection, and provided significant survival benefits, but further larger studies were needed to substantiate our findings.

Keywords: carbapenem-resistant Pseudomonas aeruginosa, ceftazidime/avibactam, polymyxin B, effectiveness, mortality

\section{Introduction}

In recent years, the rapid spread of carbapenem-resistant gram-negative bacteria (CRGNB) resistance has become a major public health concern worldwide due to the limitation of effective therapeutic options. ${ }^{1}$ Carbapenem-resistant Pseudomonas aeruginosa (CRPA), as a member of CRGNB, is listed by the World Health Organization as a "priority pathogen" requiring urgent antibacterial drug research and development. ${ }^{2}$ An epidemiological report on ICU-acquired healthcare associated infections (HAI) from the European Centre for Disease Prevention and Control (ECDC) stated that Pseudomonas aeruginosa was the most common cause of ICU-acquired pneumonia and one of the most prevalent microorganisms detected in ICU-acquired urinary tract infections and bloodstream infections. ${ }^{3}$ However, with the widespread and unreasonable use of carbapenem antibiotics, the resistance of Pseudomonas aeruginosa to these antibiotics has an upward tendency, and the total mortality rate of Pseudomonas aeruginosa infection is also at a high 
level. ${ }^{4,5}$ Currently, the treatment options for CRPA infection are limited, usually based on polymyxin (including colistin or polymyxin B), aminoglycosides, carbapenems or combination therapy, as well as novel antibiotic therapies including ceftazidime/avibactam (CAZ/AVI). ${ }^{6}$ However, polymyxin B and aminoglycoside drugs have been associated with nephrotoxic side effects, ${ }^{7}$ with increasing number of reports of Pseudomonas aeruginosa resistance to these antibiotics worldwide. ${ }^{8}$ Accordingly, it is imperative to find effective treatment approaches against CRPA infection. In this respect, novel antibacterial drugs are constantly being developed against these resistant bacteria.

Ceftazidime-Avibactam is a new $\beta$-lactam/ $\beta$-lactamase inhibitor. ${ }^{9}$ In the European Union, ceftazidime-avibactam has been approved for the treatment of adults with complicated urinary tract infections, complicated intra-abdominal infections, hospital-acquired pneumonia, and other infections caused by Gram-negative organisms in patients with limited treatment options. ${ }^{10}$ A recent study in China showed that CAZ/AVI had strong antibacterial activity against Enterobacteriaceae and Pseudomonas aeruginosa isolates collected from 30 medical centers of China Antimicrobial Surveillance Network (CHINET) in 2017. ${ }^{11}$ Likewise, studies in the United States and European countries had pointed out that, CAZ/AVI was effective against carbapenem-resistant Klebsiella pneumoniae and Pseudomonas aeruginosa in vitro, suggesting that CAZ/AVI might be an alternative treatment option for CRPA. ${ }^{12,13}$ At present, some published studies have illustrated the clinical therapeutic effects of CAZ/AVI on several different pathogens of CRGNB. ${ }^{14-16}$ Meanwhile, several studies have also displayed the benefits of CAZ/AVI in the treatment of CRGNB, with the reduced mortality to a certain extent compared with the control group. ${ }^{17-20}$

Current treatment regimens against CRPA infection included CAZ/AVI monotherapy and polymyxin-based combination treatment. Nonetheless, there were limited data on the comparison of CAZ/AVI and polymyxin in the treatment of patients with CRPA infection. For this purpose, this study was designed to compare the effectiveness of CAZ/AVI versus polymyxin B in patients with CRPA infection and to analyze the independent predictors associated with mortality and survival.

\section{Materials and Methods}

\section{Patient and Study Setting}

This was a single-center, retrospective and observational study conducted in the general intensive care unit (40 beds) of the Second Affiliated Hospital of Zhejiang University School of Medicine, a 3500-bed tertiary care teaching hospital in Hangzhou, Zhejiang in China, from January 2018 to December 2020. Patients with CRPA infection were included, and all received CAZ/AVI monotherapy or polymyxin B-based combination therapy. The usage of CAZ/AVI or polymyxin $\mathrm{B}$ was followed by the guideline recommendations during the period. The inclusion criteria consisted of (i) patients older than 16 years old, (ii) patients with monomicrobial CRPA infection diagnosed by clinicians, (iii) patients who received treatment with CAZ/AVI or polymyxin B. Exclusion criteria were as follows: patients with a duration of treatment of less than 72 hours; extremely severe burn; repeated study drug exposed. The primary outcomes were the 14-day and 30-day mortality rates. The secondary outcomes were in-hospital mortality and bacterial clearance. This study was conducted in accordance with the Declaration of Helsinki, and approved by the Research Ethics Committee of the Second Affiliated Hospital of Zhejiang University. The ethics committee waived the need for informed consent from patients due to the retrospective and anonymous characters of the study. In this study, a large amount of data was automatically collected and generated through the database to control selection bias and information bias related to the study.

\section{Data Collection}

Patients' information was obtained from the electronic medical records. The baseline data consisted of: demographic characteristics, underlying diseases and comorbidities, the use of corticosteroid and immunosuppressants, invasive procedures (including endotracheal intubation, tracheotomy, mechanical ventilation, central venous catheterization, continuous renal replacement therapy (CRRT), pulse indicator continuous cardiac output (PICCO), and extracorporeal membrane oxygenation (ECMO)) and recent surgical history. The Charlson comorbidity index (CCI) and age-adjusted Charlson comorbidity index (aCCI) were scored at admission according to the criteria, in order to quantify the comorbidity condition of patients. ${ }^{21}$ Other data were infection and treatment-related variables, including septic shock, 
the site of infection, time duration from the onset of infection to initiation of treatment, and duration of treatment. In addition, outcome variables included therapeutic outcomes (14-day, 30-day, and in-hospital mortality), length of ICU and total hospital stay, and bacterial clearance.

\section{Definitions}

Septic shock was defined in accordance with Third International Consensus Definitions for Sepsis and Septic Shock (Sepsis-3). ${ }^{22}$ The definition of pulmonary infection was based on the Infectious Diseases Society of America (IDSA) standard of American. ${ }^{23}$ Bloodstream infection was defined as the presence of at least one positive blood culture of the target pathogen accompanied by signs and symptoms of infection. The diagnosis of pulmonary infection and bloodstream infection was based on the guidelines by the (IDSA) ${ }^{23}$ Intra-abdominal infection diagnosed on the basis of the guidelines by the SIS (Surgical Infection Society) and IDSA, ${ }^{24}$ was defined as the spread of infection to the peritoneal cavity and was associated with abscess formation and the occurrence of peritonitis. Other sites of infections diagnosed according to the guidelines, ${ }^{23,25-28}$ included urinary tract infections, intracranial infections, intestinal infections, wound infections, and mediastinal infections. Bacterial clearance was confirmed when two negative cultures were obtained after treatment, and there was no recurrence within a week.

\section{Microbiology}

In this study, microorganisms were identified using the VITEK system (bioMérieux, Marcy l'Etoile, France), and susceptibility testing was performed and interpreted according to the Clinical and Laboratory Standards Institute (CLSI) guidelines. The isolates from patients with CRPA infections was tested for susceptibility to some $\beta$-lactams, aminoglycosides and quinolones antibiotics. Carbapenem resistance was defined as: the resistance to ertapenem (the minimum inhibitory concentration (MIC) $>2 \mathrm{mg} / \mathrm{mL}$ ), and the resistance to imipenem or meropenem (the MIC $>4 \mathrm{mg}$ / $\mathrm{mL}$ ) according to the CLSI guidelines. ${ }^{29}$

\section{Statistical Analysis}

Categorical variables were expressed as absolute numbers and relative frequencies, and compared using the Chi-square test or Fisher's exact test. Continuous variables were expressed as the mean \pm variance and compared using the Student's $t$-test when they followed a normal distribution. Otherwise, continuous variables if not normally distributed were expressed as the median and interquartile range (IQR), and the Mann-Whitney $U$-test was used to compare groups. The COX proportional hazard model was used to estimate the crude and adjusted hazard ratios associated with mortality. After univariate analysis, significant variables $(p<0.05)$ were entered into the multivariate regression model for analysis to identify independent risk factors for mortality. Propensity score matching method was performed to control for confounding variables in our study with propensity score estimated using logistic regression model. In this study, a one-to-one nearest neighbor matching algorithm was adopted with a caliper width of 0.02. Variables adjusted for propensity score matching in the study included: age, chronic pulmonary disease, organ transplantation, immunosuppressants, corticosteroid use, surgical history, bloodstream infections, and septic shock. The Kaplan-Meier method was used for survival analysis. All statistical analyses were performed in IBM SPSS Statistics v.25.0, and P values $<0.05$ were statistically significant.

\section{Results}

\section{Characteristics of Baseline and Microbiology}

During the study period, 136 patients with CRPA infection were eligible and enrolled in the study (Figure 1). Among them, 51 patients were treated with ceftazidime/avibactam monotherapy while 85 patients received the therapy of polymyxin B mostly combined with other antibiotics (carbapenems and amikacin etc.). Table 1 provides a comparison of the characteristics of patients receiving the treatment of CAZ/AVI or polymyxin B due to CRPA infection. In our study cohort, the overall median age was 61.5 (IQR, 51.25-70), with no significant difference between the two groups. The overall median CCI and aCCI scores were 3 (IQR, 2-4) and 4 (IQR, 3-6), respectively. The scores of the two groups were similar at baseline. However, the proportion of chronic pulmonary diseases comorbidity in the CAZ/AVI group was 


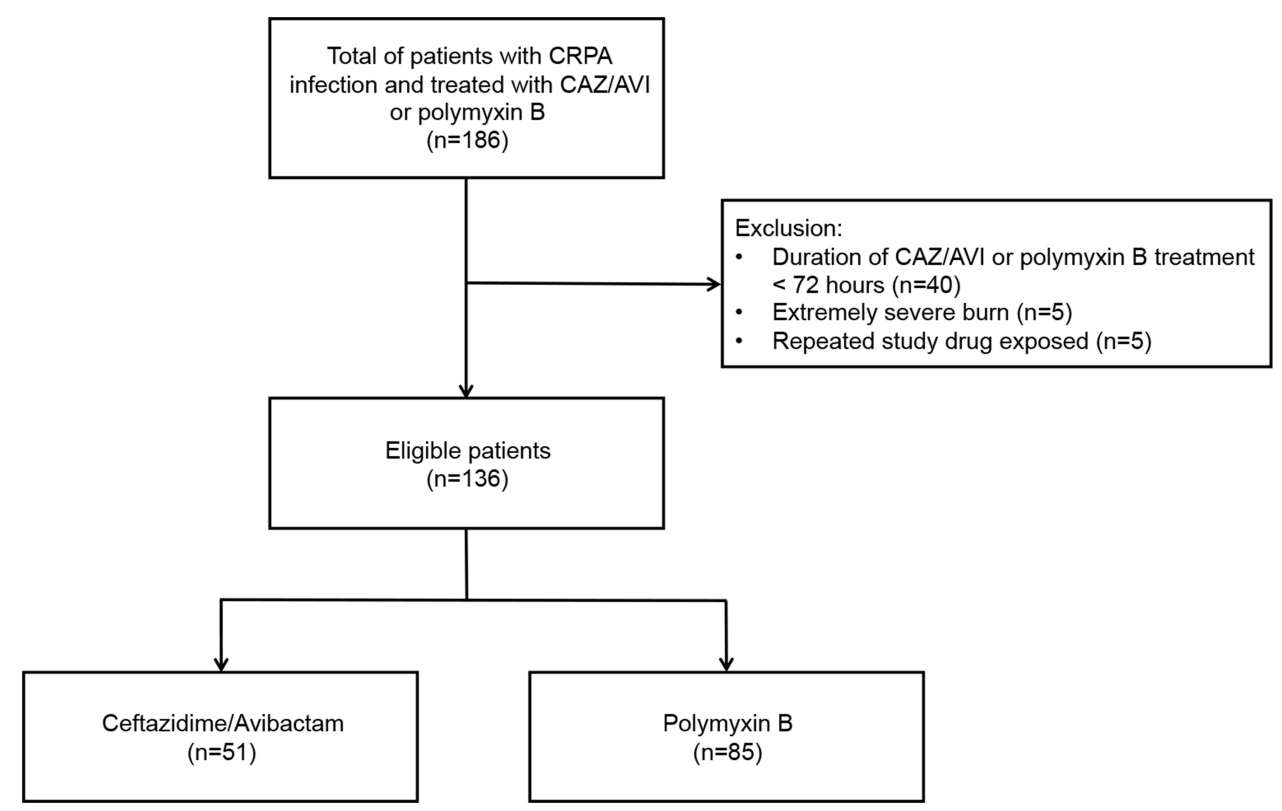

Figure I Study flow chart.

Abbreviations: CRPA, Carbapenem-resistant Pseudomonas aeruginosa; CAZ/AVI, ceftazidime/avibactam.

significantly higher than in the polymyxin B group ( $49 \%$ vs $11.8 \%, \mathrm{p}<0.001)$. In addition, the proportion of organ transplants in the CAZ/AVI group $(41.2 \%$ vs $7.1 \%, \mathrm{p}<0.001)$ was also apparently higher than that in the polymyxin $\mathrm{B}$ group, as were immunosuppressants $(43.1 \%$ vs $11.8 \%, \mathrm{p}<0.001)$ and corticosteroid $(60.8 \%$ vs $31.8 \%, \mathrm{p}=0.001)$ use. Overall, patients with a surgical history accounted for a relatively high proportion $(74.3 \%)$, with significantly more patients in the CAZ/AVI group than in the polymyxin B group $(88.2 \%$ vs $65.9 \%, \mathrm{p}=0.004)$. Furthermore, there was no significant difference in the proportion of invasive operations between the two groups, including endotracheal intubation, tracheotomy, mechanical ventilation and central venous catheterization. Regarding the severity of disease, the incidence of septic shock ( $31.4 \%$ vs $48.2 \%$, p=0.054) and CRRT (39.2\% vs $54.1 \%, \mathrm{p}=0.092)$ in the CAZ/AVI group was lower; however, there was no statistically significant difference.

In the overall study cohort, almost all patients had pulmonary infections $(99.3 \%)$, while some had bloodstream infections (36\%). Besides, about $21.3 \%$ of patients concurrently presented with other site infections, including skin and soft-tissue, urinary tract, intracranial infection etc. In the CAZ/AVI group, the proportion of pulmonary, bloodstream and other site infection was $100 \%, 23.5 \%$ and $25.5 \%$, respectively. In the polymyxin B group, the ratio of pulmonary infection (98.8\%) and other site infection (18.8\%) was similar to the CAZ/AVI group; however, nearly half of the patients had bloodstream infections (43.5\%), with a significantly higher incidence rate than in the CAZ/AVI group $(\mathrm{p}=0.019)$.

\section{Treatment Characteristics}

Treatment characteristics of patients in our study were outlined in Table 1 and details of the related antibiotics used in two groups were shown in Table S1. In addition, antimicrobial susceptibility of isolates from patients with CRPA infections in two groups was displayed in Table S2. As shown in Table 1, in the overall cohort, the median initiation time of CAZ/AVI or polymyxin B treatment was 6-7 days (IQR, 4-12.75 days), and the median duration of treatment was 910 days (IQR, 5-14 days); no statistical difference was found between the two groups. The median length of the overall ICU hospital stay was 28 days (IQR, 17-44.5 days), with a median length of 38 days (IQR, 25-74 days) and 23 days (IQR, 14-33 days) in the CAZ/AVI and polymyxin B groups, respectively. Obviously, the CAZ/AVI group had a longer ICU hospital stay than the polymyxin B group $(\mathrm{p}<0.001)$. Similarly, the total length of hospital stay was significantly longer in the CAZ/AVI group than in the polymyxin B group $(\mathrm{p}<0.001)$. 
Table I Comparison of Clinical Characteristics Between CAZ/AVI and Polymyxin B in Patients with CRPA Infection

\begin{tabular}{|c|c|c|c|c|}
\hline Characteristics & Total $(n=\mid 36)$ & CAZIAVI $(n=5 I)$ & Polymyxin B $(n=85)$ & $P$ value \\
\hline \multicolumn{5}{|l|}{ Demographic variables } \\
\hline Male sex & $100(73.5)$ & $37(72.5)$ & $63(74.1)$ & 0.841 \\
\hline Age (years) & $61.5(51.25-70)$ & $65(58-70)$ & $58(48-69)$ & 0.052 \\
\hline BMI & $23.55 \pm 4.47$ & $23.28 \pm 5.07$ & $23.7 I \pm 4.10$ & 0.588 \\
\hline $\mathrm{CCl}$ & $3(2-4)$ & $3(2-4)$ & $3(2-4)$ & 0.248 \\
\hline $\mathrm{aCCl}$ & $4(3-6)$ & $5(3-6)$ & $4(3-6)$ & 0.493 \\
\hline APACHE II score & $16(12-22)$ & $15(12-19)$ & $18(12-22)$ & 0.175 \\
\hline SOFA score & $6(4-8)$ & $5(4-7)$ & $7(4-8)$ & 0.054 \\
\hline \multicolumn{5}{|l|}{ Comorbidities } \\
\hline Cardiovascular disease & $46(33.8)$ & $17(33.3)$ & $29(34.1)$ & 0.925 \\
\hline Chronic pulmonary disease & $35(25.7)$ & $25(49.0)$ & $10(11.8)$ & $<0.001$ \\
\hline Chronic kidney disease & $7(5.1)$ & $3(5.9)$ & $4(4.7)$ & 1 \\
\hline Chronic liver disease & $9(6.6)$ & $3(5.9)$ & $6(7.1)$ & I \\
\hline Cerebrovascular disease & $39(28.7)$ & $10(19.6)$ & $29(34.1)$ & 0.070 \\
\hline Gastrointestinal diseases & $15(11.0)$ & $7(13.7)$ & $8(9.4)$ & 0.437 \\
\hline Hypertension & $57(41.9)$ & $20(39.2)$ & $37(43.5)$ & 0.622 \\
\hline Diabetes mellitus & $32(23.5)$ & $13(25.5)$ & $19(22.4)$ & 0.676 \\
\hline Solid tumor & $16(11.8)$ & $5(9.8)$ & II (12.9) & 0.582 \\
\hline Hematologic malignancy & $2(1.5)$ & $I(2.0)$ & $\mathrm{I}(\mathrm{I} .2)$ & 1 \\
\hline Organ transplant & $27(19.9)$ & $2 I(4 I .2)$ & $6(7.1)$ & $<0.001$ \\
\hline Chemotherapy & $2(1.5)$ & I (2.0) & $\mathrm{I}(\mathrm{I} .2)$ & 1 \\
\hline Immunosuppressants use & $32(23.5)$ & $22(43.1)$ & $10(11.8)$ & $<0.001$ \\
\hline Corticosteroid use & $58(42.6)$ & $31(60.8)$ & $27(31.8)$ & 0.001 \\
\hline Surgical history & I0I (74.3) & $45(88.2)$ & $56(65.9)$ & 0.004 \\
\hline \multicolumn{5}{|l|}{ Invasive procedures } \\
\hline Endotracheal intubation & $122(89.7)$ & $43(84.3)$ & $79(92.9)$ & 0.109 \\
\hline Tracheotomy & 81 (59.6) & $30(58.8)$ & $51(60.0)$ & 0.892 \\
\hline Mechanical ventilation & $136(100)$ & $51(100)$ & $85(100)$ & 1 \\
\hline Central venous catheter & II8 (86.8) & $48(94.1)$ & $70(82.4)$ & 0.050 \\
\hline CRRT & $66(48.5)$ & $20(39.2)$ & $46(54.1)$ & 0.092 \\
\hline Septic shock & $57(41.9)$ & $16(31.4)$ & $4 I(48.2)$ & 0.054 \\
\hline \multicolumn{5}{|l|}{ Infection variables } \\
\hline Pulmonary infection & $135(99.3)$ & $51(100)$ & $84(98.8)$ & I \\
\hline Bloodstream infection & $49(36.0)$ & $12(23.5)$ & $37(43.5)$ & 0.019 \\
\hline Intra-abdominal infection & $4(2.9)$ & $0(0)$ & $4(4.7)$ & 0.294 \\
\hline Other infection sites & $29(21.3)$ & $13(25.5)$ & $16(18.8)$ & 0.358 \\
\hline \multicolumn{5}{|l|}{ Treatment variables } \\
\hline Duration of therapy & $9(5-14)$ & $10(6-17)$ & $9(5-12)$ & 0.108 \\
\hline Time to initiation of treatment & $6.5(4-12.75)$ & $6(4-15)$ & $7(3.5-10.5)$ & 0.580 \\
\hline \multicolumn{5}{|l|}{ Outcomes } \\
\hline The length of ICU (days) & $28(17-44.5)$ & $38(25-74)$ & $23(14-33)$ & $<0.001$ \\
\hline The length of hospitals (days) & $34(22.67-6 I)$ & $48(33-83)$ & $29(19-43)$ & $<0.001$ \\
\hline I4-day mortality & $26(19.1)$ & $3(5.9)$ & $23(27.1)$ & 0.002 \\
\hline 30-day mortality & $47(34.6)$ & $7(13.7)$ & $40(47.1)$ & $<0.001$ \\
\hline In-hospital mortality & $66(48.5)$ & $15(29.4)$ & $51(60.0)$ & 0.001 \\
\hline Bacterial eradication & $34(25.0)$ & $23(45.1)$ & II (12.9) & $<0.001$ \\
\hline
\end{tabular}

Notes: Data are expressed as number (\%) or mean \pm SD or median (IQR).

Abbreviations: CAZ/AVI, Ceftazidime/avibactam; BMI, Body mass index; CCl, Charlson comorbidity index; aCCl, age-adjusted Charlson comorbidity index; CRRT, Continuous renal replacement therapy; ICU, Intensive care unit; IQR, Interquartile range. 


\section{Outcomes}

Regarding primary outcomes (Table 1 ), the 14 -day mortality rate ( 5.9 vs $27.1 \%, \mathrm{p}=0.002)$ and the 30 -day mortality rate $(13.7 \%$ vs $47.1 \%, p<0.001)$ in the CAZ/AVI group were significantly lower than in the polymyxin B group. Meanwhile, the difference in in-hospital mortality between the two groups was also significant $(29.4 \%$ vs $60.0 \%, p=0.001)$. Furthermore, a higher frequency of bacterial eradication was observed with the use of CAZ/AVI compared with polymyxin B (45.1\% vs $12.9 \%, \mathrm{p}<0.001)$. In addition, after adjusting some variables using the propensity matching scoring method to control the consistency of baseline levels between the two groups, we compared the outcome variables of the CAZ/AVI group with polymyxin B group (Table 2), and found that the 30-day mortality rate of CAZ/AVI treatment was still significantly lower than that of the polymyxin B group $(14.3 \%$ vs $42.9 \%, p=0.018)$, but there was no statistical difference in 14 -day mortality rate $(7.1 \%$ vs $25 \%, \mathrm{p}=0.146)$ and in-hospital mortality $(35.7 \%$ vs $53.6 \%$, $\mathrm{p}=0.179)$. Besides, the bacterial clearance rate in the CAZ/AVI group was higher than in the polymyxin B group (42.9\% vs $14.3 \%, p=0.018$ ); however, the CAZ/AVI group had longer ICU hospital stay $(\mathrm{p}=0.021)$ and total hospital stay $(\mathrm{p}=0.008)$ compared with the polymyxin B group.

\section{Independent Predictors of Mortality}

Characteristics of the survivor and non-survivor sub-groups at 30 days were described in Table 3. Significant differences in CCI ( $\mathrm{p}=0.015)$, aCCI $(\mathrm{p}=0.045)$, chronic pulmonary disease $(\mathrm{p}=0.003)$, organ transplant $(\mathrm{p}=0.016)$, surgical history $(\mathrm{p}=0.004)$, tracheotomy $(\mathrm{p}=0.010)$, CRRT treatment $(\mathrm{p}<0.001)$, septic shock $(\mathrm{p}<0.001)$ were observed. Table 4 showed the univariate and multivariate analysis of factors related to 30-day mortality. During the multivariate COX regression analysis (Table 4), CRRT and septic shock were independent predictors of 30-day mortality while CAZ/AVI therapy, surgical history and the duration of therapy were independent predictors of 30-day survival. After adjustment by propensity score matching, the adjusted multivariate COX analysis showed (Table 5) that the age was identified as independent predictor of 30-day mortality while CAZ/AVI therapy and central venous catheterization emerged as independent predictors of 30-day survival. The Kaplan-Meier curves of patients who received CAZ/AVI or polymyxin $B$ for the treatment of CRPA infection were depicted in Figure $2(p<0.001)$. The survival curve analysis visually showed that the risk of mortality in CAZ/AVI treatment for patients with CRPA infection was reduced.

\section{Discussion}

This was a single-center retrospective observational cohort study of critically ill patients with CRPA infection treated with CAZ/AVI or polymyxin B. Importantly, we found that CAZ/AVI therapy provided a significant survival benefit compared to polymyxin B therapy in patients with CRPA infection; patients treated with CAZ/AVI had lower 14-day, 30day and in-hospital mortality than those treated with polymyxin B. After adjusting for confounders, the 30-day mortality in the CAZ/AVI group was still significantly lower than that in the polymyxin B group. In terms of bacterial clearance, the clearance rate of the CAZ/AVI group was obviously higher than polymyxin B group, even after the adjustment of PSM. Besides, the adjusted multivariate COX analysis showed that the age was identified as independent predictor of 30day mortality while CAZ/AVI therapy and central venous catheterization were independent predictors of 30-day survival.

Based on the above analysis results, CAZ/AVI was demonstrated to effectively treat patients with CRPA infection in this cohort. In terms of outcome variables, this study showed the clinical efficacy of CAZ/AVI in the treatment of CRPA infection, characterized by a lower in-hospital mortality rate (29.4\%), and a lower mortality rate at 14 and 30 days $(5.9 \%, 13.7 \%$, respectively) and a higher bacterial clearance rate $(45.1 \%)$. A retrospective study designed to evaluate and describe the characteristics and outcomes of 8 patients with multidrug-resistant (MDR) and extensively drugresistant (XDR) Pseudomonas aeruginosa infections treated with CAZ/AVI showed that the 30-day mortality rate was $12.5 \%$, which was consistent with our findings. ${ }^{16}$ Another multi-center retrospective study was conducted in 6 medical centers in the United States from 2015 to 2019, enrolling a total of 203 subjects with carbapenem-resistant Enterobacteriaceae $(\mathrm{n}=117)$ and Pseudomonas aeruginosa $(\mathrm{n}=63)$ isolated from culture specimens. The 30-day mortality rate after CAZ/AVI treatment was $17.2 \%$, which was also in line with our findings. ${ }^{19}$ These studies shown that CAZ/ AVI had apparent clinical effects on patients with CRPA infection, and the potential reason might be that CAZ/AVI had 
Table 2 Comparison of Clinical Characteristics Between CAZ/AVI and Polymyxin B in Patients with CRPA Infection After Adjustment

\begin{tabular}{|c|c|c|c|c|}
\hline Characteristics & Total $(n=56)$ & CAZIAVI $(n=28)$ & Polymyxin B $(n=28)$ & $P$ value \\
\hline \multicolumn{5}{|l|}{ Demographic variables } \\
\hline Male sex & $42(75.0)$ & $20(7 \mid .4)$ & $22(78.6)$ & 0.537 \\
\hline Age (years) & $62.5(48.25-70.75)$ & $62.5(58-70)$ & $62.5(47.25-7 I)$ & 0.780 \\
\hline BMI & $23.24 \pm 4.02$ & $23.84 \pm 4.77$ & $22.63 \pm 3.07$ & 0.264 \\
\hline $\mathrm{CCl}$ & $3(2-4)$ & $3(2-3)$ & $3(1.25-5)$ & 0.363 \\
\hline $\mathrm{aCCl}$ & $4.70 \pm 2.51$ & $4.57 \pm 2.22$ & $4.82 \pm 2.80$ & 0.713 \\
\hline APACHE II score & $16(12.25-20)$ & I5.5 (14-24.25) & $16(10.5-19.5)$ & 0.485 \\
\hline SOFA score & $6(4-8)$ & $6(4-8)$ & $6(4-8)$ & 0.954 \\
\hline \multicolumn{5}{|l|}{ Comorbidities } \\
\hline Cardiovascular disease & $17(30.4)$ & $9(32.1)$ & $8(28.6)$ & 0.771 \\
\hline Chronic pulmonary disease & II (19.6) & $6(21.4)$ & $5(17.9)$ & 0.737 \\
\hline Chronic kidney disease & $5(8.9)$ & $3(10.7)$ & $2(7.1)$ & I \\
\hline Chronic liver disease & $5(8.9)$ & I (3.6) & $4(14.3)$ & 0.349 \\
\hline Cerebrovascular disease & $16(28.6)$ & $8(28.6)$ & $8(28.6)$ & I \\
\hline Gastrointestinal diseases & $5(8.9)$ & $2(7.1)$ & $3(10.7)$ & 1 \\
\hline Hypertension & $23(4 I .1)$ & II (39.3) & $12(42.9)$ & 0.786 \\
\hline Diabetes mellitus & II (19.6) & $7(25.0)$ & $4(14.3)$ & 0.313 \\
\hline Solid tumor & $8(14.3)$ & $3(10.7)$ & $5(17.9)$ & 0.703 \\
\hline Hematologic malignancy & I (I.8) & I (3.6) & $0(0.0)$ & I \\
\hline Organ transplant & $10(17.9)$ & $5(17.9)$ & $5(17.9)$ & I \\
\hline Chemotherapy & I (I.8) & I (3.6) & $0(0.0)$ & 1 \\
\hline Immunosuppressants use & II (19.6) & $6(21.4)$ & $5(17.9)$ & 0.737 \\
\hline Corticosteroid use & $27(48.2)$ & $14(50.0)$ & $13(46.4)$ & 0.789 \\
\hline Surgical history & $46(82.1)$ & $22(78.6)$ & $24(85.7)$ & 0.485 \\
\hline \multicolumn{5}{|l|}{ Invasive procedures } \\
\hline Endotracheal intubation & $50(89.3)$ & $23(82.1)$ & $27(96.4)$ & 0.195 \\
\hline Tracheotomy & $37(66.1)$ & $19(67.9)$ & $18(64.3)$ & 0.778 \\
\hline Mechanical ventilation & $56(100)$ & $28(100)$ & $28(100)$ & I \\
\hline Central venous catheter & $50(89.3)$ & $26(92.9)$ & $24(85.7)$ & 0.666 \\
\hline CRRT & $27(48.2)$ & $12(42.9)$ & $15(53.6)$ & 0.422 \\
\hline Septic shock & $25(44.6)$ & $13(46.4)$ & $12(42.9)$ & 0.788 \\
\hline \multicolumn{5}{|l|}{ Infection variables } \\
\hline Pulmonary infection & $55(98.2)$ & $28(100)$ & $27(96.4)$ & 1 \\
\hline Bloodstream infection & $18(32.1)$ & $10(35.7)$ & $8(28.6)$ & 0.567 \\
\hline Intra-abdominal infection & $2(3.6)$ & $0(0)$ & $2(7.1)$ & 0.471 \\
\hline Other infection sites & $15(26.8)$ & $9(32.1)$ & $6(21.4)$ & 0.365 \\
\hline \multicolumn{5}{|l|}{ Treatment variables } \\
\hline Duration of therapy & $9(5.25-14.75)$ & $10(6-16.75)$ & $8.5(5-11)$ & 0.109 \\
\hline Time to initiation of treatment & $7(4-14.5)$ & $10.5(4-18)$ & $6(4-10.5)$ & 0.366 \\
\hline \multicolumn{5}{|l|}{ Outcomes } \\
\hline The length of ICU (days) & $28(17-43.75)$ & $32.5(19.52-72)$ & $24.5(14-32.5)$ & 0.021 \\
\hline The length of hospitals (days) & $33.5(24.3-60.5)$ & $46(27.5-78.5)$ & $29.5(19-43.25)$ & 0.008 \\
\hline I4-d mortality & $9(16.1)$ & $2(7.1)$ & $7(25.0)$ & 0.146 \\
\hline 30-d mortality & $16(28.6)$ & $4(14.3)$ & $12(42.9)$ & 0.018 \\
\hline In-hospital mortality & $25(44.6)$ & $10(35.7)$ & $15(53.6)$ & 0.179 \\
\hline Bacterial eradication & $16(28.6)$ & $12(42.9)$ & $4(14.3)$ & 0.018 \\
\hline
\end{tabular}

Notes: Data are expressed as number (\%) or mean \pm SD or median (IQR).

Abbreviations: CAZ/AVI, Ceftazidime/avibactam; BMI, Body mass index; CCl, Charlson comorbidity index; aCCl, age-adjusted Charlson comorbidity index; CRRT, Continuous renal replacement therapy; ICU, Intensive care unit; IQR, Interquartile range. 
Table 3 Comparison of Clinical Characteristics Between 30-Day Survivors and Non-Survivors with CRPA Infection

\begin{tabular}{|c|c|c|c|c|}
\hline Characteristics & Total $(n=136)$ & Survivors $(n=89)$ & Non-Survivors $(n=47)$ & $\mathbf{P}$ value \\
\hline \multicolumn{5}{|l|}{ Demographic variables } \\
\hline Male sex & $100(73.5)$ & $67(75.3)$ & $33(70.2)$ & 0.524 \\
\hline Age (years) & $61.5(51.25-70)$ & $62(50.5-70.0)$ & $61(53-7 \mid)$ & 0.980 \\
\hline $\mathrm{BMI}$ & $23.55 \pm 4.47$ & $23.76 \pm 4.67$ & $23.15 \pm 4.10$ & 0.446 \\
\hline $\mathrm{CCl}$ & $3(2-4)$ & $3(1-4)$ & $3(3-4)$ & 0.015 \\
\hline $\mathrm{aCCl}$ & $4(3-6)$ & $4(3-6)$ & $5(4-6)$ & 0.045 \\
\hline APACHE II score & $16(12-22)$ & $16(12-20)$ & $17(12-23)$ & 0.277 \\
\hline SOFA score & $6(4-8)$ & $6(4-8)$ & $7(4-9)$ & 0.338 \\
\hline \multicolumn{5}{|l|}{ Comorbidities } \\
\hline Cardiovascular disease & $46(33.8)$ & $33(37.1)$ & I3 (27.7) & 0.270 \\
\hline Chronic pulmonary disease & $35(25.7)$ & $30(33.7)$ & $5(10.6)$ & 0.003 \\
\hline Chronic kidney disease & $7(5.1)$ & $5(5.6)$ & $2(4.3)$ & 1 \\
\hline Chronic liver disease & $9(6.6)$ & $7(7.9)$ & $2(4.3)$ & 0.658 \\
\hline Cerebrovascular disease & $39(28.7)$ & $23(25.8)$ & $16(34.0)$ & 0.315 \\
\hline Gastrointestinal diseases & $15(11.0)$ & $10(11.2)$ & $5(10.6)$ & 0.916 \\
\hline Hypertension & $57(41.9)$ & $38(42.7)$ & $19(40.4)$ & 0.799 \\
\hline Diabetes mellitus & $32(23.5)$ & $19(21.3)$ & $13(27.7)$ & 0.409 \\
\hline Solid tumor & $16(11.8)$ & $9(10.1)$ & $7(14.9)$ & 0.411 \\
\hline Hematologic malignancy & $2(1.5)$ & $\mathrm{I}(\mathrm{I} . \mathrm{I})$ & $\mathrm{I}(2 . \mathrm{I})$ & 1 \\
\hline Organ transplant & $27(19.9)$ & $23(25.8)$ & $4(8.5)$ & 0.016 \\
\hline Chemotherapy & $2(1.5)$ & $\mathrm{I}(\mathrm{I} . \mathrm{I})$ & $\mathrm{I}(2.1)$ & I \\
\hline Immunosuppressants use & $32(23.5)$ & $23(25.8)$ & $9(19.1)$ & 0.381 \\
\hline Corticosteroid use & $58(42.6)$ & $37(41.6)$ & $21(44.7)$ & 0.727 \\
\hline Surgical history & $101(74.3)$ & $73(82.0)$ & $28(59.6)$ & 0.004 \\
\hline \multicolumn{5}{|l|}{ Invasive procedures } \\
\hline Endotracheal intubation & $122(89.7)$ & $78(87.6)$ & $44(93.6)$ & 0.427 \\
\hline Tracheotomy & $81(59.6)$ & $60(67.4)$ & $21(44.7)$ & 0.010 \\
\hline Mechanical ventilation & $136(100)$ & $89(100)$ & $47(100)$ & I \\
\hline Central venous catheter & $118(86.8)$ & $77(86.5)$ & $4 \mid(87.2)$ & 0.907 \\
\hline CRRT & $66(48.5)$ & $30(33.7)$ & $36(76.6)$ & $<0.001$ \\
\hline Septic shock & $57(41.9)$ & $25(28.1)$ & $32(68.1)$ & $<0.001$ \\
\hline \multicolumn{5}{|l|}{ Infection variables } \\
\hline Pulmonary infection & $135(99.3)$ & $88(98.9)$ & $47(100.0)$ & 1 \\
\hline Bloodstream infection & $49(36.0)$ & $28(31.5)$ & $2 \mathrm{I}(44.7)$ & 0.127 \\
\hline Intra-abdominal infection & $4(2.9)$ & $2(2.2)$ & $2(4.3)$ & 0.900 \\
\hline Other infection sites & $29(21.3)$ & $24(27.0)$ & $5(10.6)$ & 0.027 \\
\hline \multicolumn{5}{|l|}{ Treatment variables } \\
\hline Duration of therapy & $9(5-14)$ & II (7-17) & $6(4-9)$ & $<0.001$ \\
\hline Time to initiation of treatment & $6.5(4-12.75)$ & $6(4-15)$ & $7(3-10)$ & 0.348 \\
\hline CAZ/AVI use & $51(37.5 \%)$ & $44(49.4)$ & $7(14.9)$ & $<0.001$ \\
\hline Polymyxin B use & 85 (62.5\%) & $45(50.6)$ & $40(85.1)$ & $<0.001$ \\
\hline \multicolumn{5}{|l|}{ Outcomes } \\
\hline The length of ICU (days) & $28(17-44.5)$ & $34.09(24.5-58)$ & $18(13-25)$ & $<0.001$ \\
\hline The length of hospitals (days) & $34(22.67-61)$ & $46(30-78.5)$ & $21(15-33)$ & $<0.001$ \\
\hline Bacterial eradication & $34(25.0)$ & $34(38.2)$ & $0(0)$ & $<0.001$ \\
\hline
\end{tabular}

Notes: Data are expressed as number (\%) or mean \pm SD or median (IQR).

Abbreviations: $\mathrm{BMI}$, Body mass index; $\mathrm{CCl}$, Charlson comorbidity index; aCCl, age-adjusted Charlson comorbidity index; CRRT, Continuous renal replacement therapy; ICU, Intensive care unit; IQR, Interquartile range.

a good activity against CRPA. A CHINET report in 2017 showed that $86.5 \%$ of CRPA isolates were susceptible to CAZ/ AVI in vitro. ${ }^{11}$ Meanwhile, another study by Alatoom et al demonstrated the sensitivity of 29 strains (94\%) of Pseudomonas aeruginosa to CAZ/AVI $\left(\mathrm{MIC}_{50} 1.5 \mu \mathrm{g} / \mathrm{mL}\right) .{ }^{30} \mathrm{In}$ contrast, a study collecting Gram-negative clinical isolates from 45 medical centers across China in the CHINET Program in 2018, mentioned that the resistance rate of PA 
Table 4 Univariate and Multivariate COX Regression Analysis Associated with 30-Day Mortality

\begin{tabular}{|l|l|l|l|c|}
\hline \multirow{2}{*}{ Variables } & \multicolumn{2}{|c|}{ Univariate Analysis } & \multicolumn{2}{c|}{ Multivariate Analysis } \\
\cline { 2 - 5 } & P value & HR (95\% CI) & P value & HR (95\% CI) \\
\hline CCI (I-point increments) & 0.040 & $I .179(I .008-1.379)$ & & \\
Chronic pulmonary disease & 0.012 & $0.302(0.120-0.765)$ & & \\
Organ transplant & 0.035 & $0.332(0.119-0.926)$ & & \\
Surgical history & 0.002 & $0.389(0.217-0.698)$ & 0.026 & $0.506(0.277-0.923)$ \\
Tracheotomy & 0.004 & $0.425(0.239-0.757)$ & & \\
CRRT & $<0.001$ & $4.46 I(2.266-8.786)$ & 0.003 & $3.159(1.479-6.745)$ \\
Septic shock & $<0.001$ & $3.739(2.020-6.919)$ & 0.030 & $2.133(1.078-4.222)$ \\
Other infection sites & 0.028 & $0.353(0.140-0.894)$ & & \\
Duration of therapy (I-day increments) & $<0.00 I$ & $0.847(0.785-0.913)$ & $<0.001$ & $0.853(0.794-0.917)$ \\
CAZ/AVI use & $<0.00 I$ & $0.235(0.105-0.525)$ & 0.028 & $0.394(0.172-0.902)$ \\
\hline
\end{tabular}

Abbreviations: HR, Hazard ratios; Cl, Confidence interval; $\mathrm{CCl}$, Charlson comorbidity index; CRRT, Continuous renal replacement therapy; CAZ/AVI, Ceftazidime/avibactam.

Table 5 Univariate and Multivariate COX Regression Analysis Associated with 30-Day Mortality After Adjustment

\begin{tabular}{|l|l|l|l|c|}
\hline \multirow{2}{*}{} & \multicolumn{2}{|c|}{ Univariate Analysis } & \multicolumn{2}{c|}{ Multivariate Analysis } \\
\cline { 2 - 5 } & P value & HR (95\% CI) & P value & HR (95\% Cl) \\
\hline Age (I-year increments) & 0.033 & $1.047(I .004-I .093)$ & 0.022 & $1.050(1.007-I .094)$ \\
Central venous catheter & $0.02 I$ & $0.262(0.084-0.816)$ & 0.027 & $0.268(0.084-0.859)$ \\
Duration of therapy (I-day increments) & 0.027 & $0.87 I(0.77 I-0.984)$ & & \\
CAZIAVI use & 0.031 & $0.287(0.092-0.890)$ & 0.016 & $0.244(0.078-0.765)$ \\
\hline
\end{tabular}

Abbreviations: HR, Hazard ratios; Cl, Confidence interval; CAZIAVI, Ceftazidime/avibactam.

(657 isolates) to polymyxin B was $3.8 \%$, and that of CRPA (171 isolates) to polymyxin B was $5.3 \%$ in vitro. ${ }^{31}$ The CHINET surveillance (http://www.chinets.com) also revealed that, from 2019 to 2021, the resistance rates to polymyxin B were $0.8 \%, 0.7 \%$ and $1.3 \%$ respectively while the resistance rates to colistin were $1.1 \%, 2.0 \%$ and $1.6 \%$ respectively. Another retrospective study collecting 71880 PA isolates in Zhejiang Province during 2015-2017, showed that PA isolates were highly sensitive to polymyxin B and colistin (98.50\% and $99.07 \%$ respectively) in our region. ${ }^{32}$ The above data indicated that the factor of polymyxin B resistance was less likely to disturb the outcome. Accordingly, CAZ/AVI therapy showed good antibacterial effects in vitro and in vivo, providing a more effective treatment option for patients with CRPA infection.

In this study, it was observed that compared with the polymyxin B group, patients treated with CAZ/AVI for CRPA infection had longer ICU hospital stays and overall hospital stays, which might be due to the fact that the incidence of bloodstream infection and the mortality of 14-day and 30-day in the polymyxin B group were apparently higher than those in the CAZ/AVI group, leading to differences in survival time between the two groups, thus resulting in significant differences in length of hospital stay. In addition, compared with the polymyxin B group, there was a higher proportion of immunocompromised patients (transplantation, malignancy, immunosuppressant and corticosteroids use, etc) in the CAZ/ AVI group. In view of the delayed course of immunosuppressive patients, it might be one of the reasons. Therefore, it was shown that patients with CRPA infection treated with CAZ/AVI had a longer hospital stay.

In this study, the characteristics of the survivors and non-survivors at 30 days were compared to find potential independent predictors. Multivariate COX analysis showed that the age was identified as independent predictor of 30-day mortality while CAZ/AVI therapy and central venous catheterization emerged as independent predictors of 30-day survival. A multicenter retrospective study by Tumbarello et al was conducted in adult patients infected with KPC- 


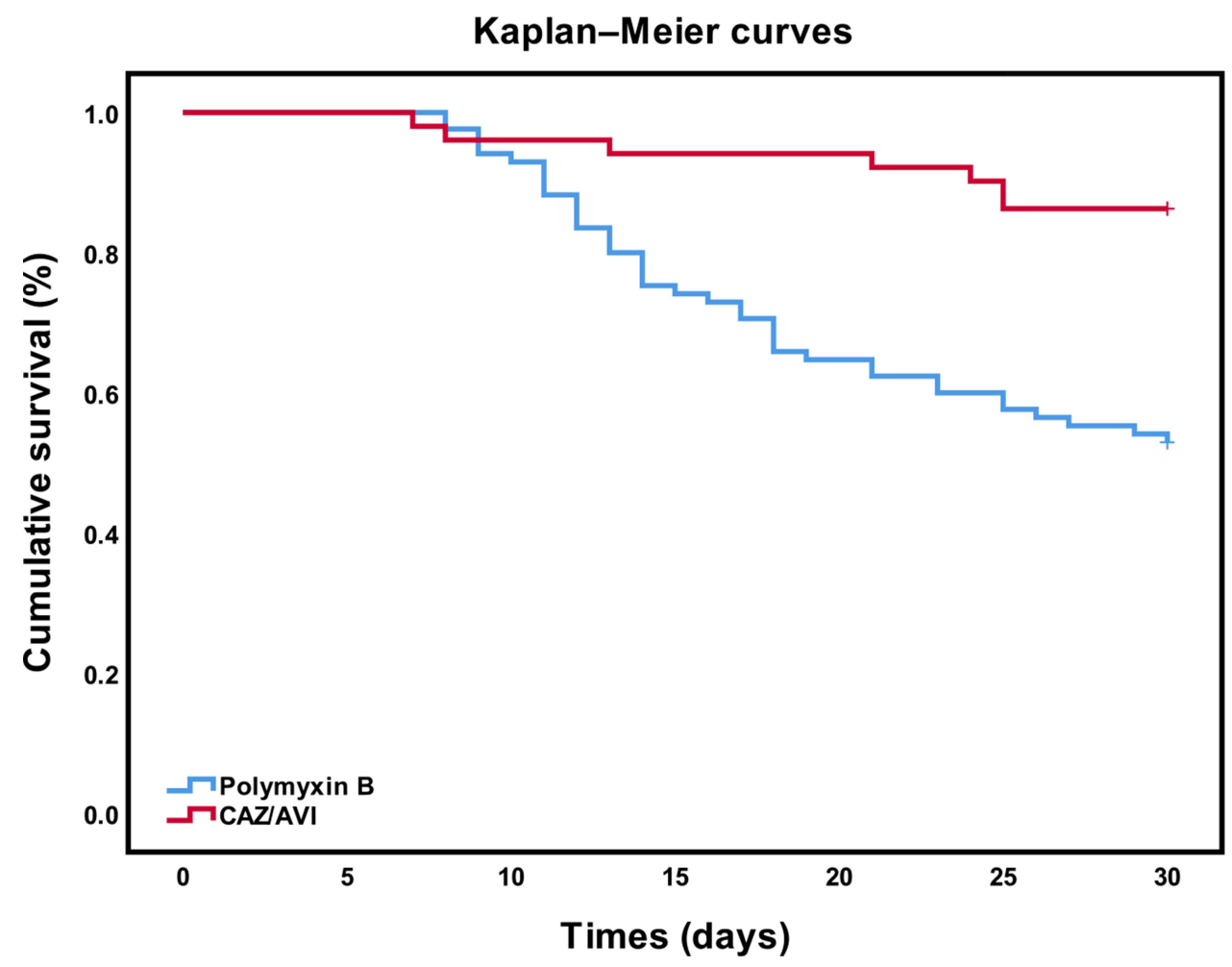

Figure 2 Kaplan-Meier curves of patients with CRPA infection treated with CAZ/AVI or polymyxin B. Abbreviations: CRPA, Carbapenem-resistant Pseudomonas aeruginosa; CAZ/AVI, ceftazidime/avibactam.

producing K. pneumoniae and treated with CAZ/AVI salvage treatment. The results of related multivariate analysis showed that septic shock, neutropenia, Charlson comorbidity index $\geq 3$ and recent mechanical ventilation were independent predictors of 30-day mortality, while receiving CAZ/AVI treatment was the only independent predictor of survival. ${ }^{33}$ The independent predictor of survival was consistent with our findings. In another retrospective study of patients with CRKP bacteremia who received CAZ/AVI for more than three days, the multivariate analysis also concluded that CAZ/AVI was an independent predictor of clinical success. ${ }^{17}$ Therefore, it could be inferred from the above findings that CAZ/AVI therapy was an effective, and alternative treatment for CRPA infection.

CAZ/AVI is a novel antimicrobial agent that can be used as a treatment option for severe infections caused by carbapenem-resistant gram-negative bacteria. ${ }^{34}$ It was approved by the US Food and Drug Administration (FDA) in 2015 and was the first combination of $\beta$-lactams to cover extensively drug-resistant gram-negative pathogens including Pseudomonas aeruginosa. ${ }^{35}$ Interestingly, data from real-world studies showed that CAZ/AVI therapy was superior to polymyxin-based treatment in the fight against CRE from the perspective of efficacy and safety. ${ }^{36}$ However, currently available data on the outcomes of CRPA infection treated with CAZ/AVI is limited, as few clinical studies sought to observe and compare the efficacy of treatment between CAZ/AVI and polymyxin against CRPA infection. Notwithstanding that the effectiveness of CAZ/AVI therapy with polymyxin has been contrasted in several studies, the infectious pathogens were somewhat different. A study by van Duin et al provided evidence of the superiority of ceftazidime-avibactam over colistin in the initial treatment of infections caused by K. pneumoniae carbapenemaseproducing carbapenem-resistant Enterobacteriaceae (CRE), indicating that the use of CAZ/AVI was associated with improved clinical outcomes, especially decreasing of all-cause hospital mortality and improving benefit-risk outcomes, ${ }^{18}$ but the cohort population were patients with CRE (mainly CRKP) infection. In a study by Hakeam et al, patients with CRE infection experienced a lower 14-day mortality rate with CAZ/AVI than with colistin. ${ }^{37}$ Another retrospective study was also conducted in patients with CRKP bacteremia, and it was observed that CAZ/AVI treatment had a higher clinical success rate and survival rate than other treatment regimens (including colistin treatment). ${ }^{17}$ To the best of our knowledge, this is the first real-world study designed to compare the clinical efficiency between CAZ/AVI and polymyxin B in 
patients with CRPA infection. In summary, our study found that CAZ/AVI treatment was superior to polymyxin B therapy in patients with CRPA infection with lower 14-and 30-day mortality and in-hospital mortality and higher bacterial clearance rate compared with polymyxin B treatment, validating the survival benefits of CAZ/AVI therapy in patients with CRPA infection.

There were many limitations in this study. First of all, this study was a single-center study and the sample size obtained was insufficient. However, it should be borne in mind that our sample size was relatively small since CAV/AVI was marketed in China in 2019. Moreover, due to the observational nature of our study, the generalizability of our study results was limited. Furthermore, our study did not include relevant indicators of the study drug sensitivity test for infectious pathogens, and details were not available.

\section{Conclusion}

This single-center observational study provided valuable information on the efficacy of two important antimicrobial agents and risk factors of mortality in patients with CRPA infection. Herein, we provided evidence that CAZ/AVI therapy was superior to polymyxin B therapy for patients with CRPA infection in the real world, demonstrating significant survival benefits for CRPA-infected patients. However, larger study populations and well-designed randomized clinical trials are needed to corroborate our findings.

\section{Abbreviations}

CAZ/AVI, Ceftazidime/avibactam; CRPA, Carbapenem-resistant Pseudomonas aeruginosa; PSM, Propensity score matching; CRGNB, Carbapenem-resistant gram-negative bacteria; HAI, Healthcare associated infections; ECDC, European Centers for Disease Control and Prevention; CHINET, China Antimicrobial Surveillance Network; CRRT, Continuous renal replacement therapy; PICCO, Pulse indicator continuous cardiac output; ECMO, Extracorporeal membrane oxygenation; CCI, Charlson comorbidity index; aCCI, age-adjusted Charlson comorbidity index; IDSA, Infectious Diseases Society of America; CLSI, Clinical and Laboratory Standards Institute; MIC, Minimum inhibitory concentration; IQR, Interquartile range; MDR, Multidrug-resistant; XDR, Extensively drug-resistant; SOT, solid organ transplantation; HSCT, Hematopoietic stem cell transplantation; CDI, Clostridium difficile infection; FDA, Food and Drug Administration; CRE, Carbapenem-resistant Enterobacteriaceae.

\section{Data Sharing Statement}

The datasets used and/or analyzed during the current study are available from the corresponding author on reasonable request.

\section{Ethics Approval}

This study was approved by the Research Ethics Committee of Second Affiliated Hospital of Zhejiang University. In addition, for organ transplantation, all organs were donated voluntarily with written informed consent, and the organ donations and transplants were conducted in accordance with the Declaration of Istanbul.

\section{Acknowledgments}

We wish to thank the staff of the general intensive care unit of the Second Affiliated Hospital of Zhejiang University for their support and cooperation in data access and analysis.

\section{Author Contributions}

All authors made a significant contribution to the work reported, whether that is in the conception, study design, execution, acquisition of data, analysis and interpretation, or in all these areas; took part in drafting, revising or critically reviewing the article; gave final approval of the version to be published; have agreed on the journal to which the article has been submitted; and agree to be accountable for all aspects of the work. 


\section{Funding}

None funding.

\section{Disclosure}

The authors declare no conflicts of interest.

\section{References}

1. Babiker A, Clarke LG, Saul M, et al. Changing epidemiology and decreased mortality associated with Carbapenem-resistant Gram-negative bacteria from 2000-2017. Clin Infect Dis. 2020. doi:10.1093/cid/ciaa1464

2. Tacconelli E, Carrara E, Savoldi A, et al. Discovery, research, and development of new antibiotics: the WHO priority list of antibiotic-resistant bacteria and tuberculosis. Lancet Infect Dis. 2018;18(3):318-327. doi:10.1016/s1473-3099(17)30753-3

3. European Centre for Disease Prevention and Control. Healthcare-associated infections acquired in intensive care units. In: ECDC. Annual epidemiological report for 2016. Stockholm: ECDC; 2018.

4. Hu Y, Liu C, Wang Q, et al. Emergence and expansion of a carbapenem-resistant pseudomonas aeruginosa clone are associated with plasmid-borne bla (KPC-2) and virulence-related genes. mSystems. 2021;6(3). doi:10.1128/mSystems.00154-21

5. Fujitani S, Sun HY, Yu VL, Weingarten JA. Pneumonia due to Pseudomonas aeruginosa: part I: epidemiology, clinical diagnosis, and source. Chest. 2011;139(4):909-919. doi:10.1378/chest.10-0166

6. Karakonstantis S, Kritsotakis EI, Gikas A. Treatment options for K. pneumoniae, P. aeruginosa and A. baumannii co-resistant to carbapenems, aminoglycosides, polymyxins and tigecycline: an approach based on the mechanisms of resistance to carbapenems. Infection. 2020;48(6):835-851. doi:10.1007/s15010-020-01520-6

7. Tuon FF, Aragao BZ, Santos TA, Gasparetto J, Cordova K, Abujamra M. Acute kidney injury in patients using amikacin in an era of carbapenem-resistant bacteria. Infect Dis. 2016;48(11-12):869-871. doi:10.1080/23744235.2016.1205215

8. Karakonstantis S, Kritsotakis EI, Gikas A. Pandrug-resistant Gram-negative bacteria: a systematic review of current epidemiology, prognosis and treatment options. J Antimicrob Chemother. 2020;75(2):271-282. doi:10.1093/jac/dkz401

9. van Duin D, Bonomo RA. Ceftazidime/avibactam and ceftolozane/tazobactam: second-generation $\beta$-lactam/ $\beta$-lactamase inhibitor combinations. Clin Infect Dis. 2016;63(2):234-241. doi:10.1093/cid/ciw243

10. Falcone M, Paterson D. Spotlight on ceftazidime/avibactam: a new option for MDR Gram-negative infections. J Antimicrob Chemother. 2016;71 (10):2713-2722. doi:10.1093/jac/dkw239

11. Yin D, Wu S, Yang Y, et al. Results from the China Antimicrobial Surveillance Network (CHINET) in 2017 of the in vitro activities of ceftazidime-avibactam and ceftolozane-tazobactam against clinical isolates of enterobacteriaceae and Pseudomonas aeruginosa. Antimicrob Agents Chemother. 2019;63(4). doi:10.1128/aac.02431-18

12. Nichols WW, De Jonge BLM, Kazmierczak KM, Karlowsky JA, Sahm DF. In vitro susceptibility of global surveillance isolates of pseudomonas aeruginosa to ceftazidime-avibactam (INFORM 2012 to 2014). Antimicrob Agents Chemother. 2016;60(8):4743-4749. doi:10.1128/aac.00220-16

13. Testa R, Cantón R, Giani T, et al. In vitro activity of ceftazidime, ceftaroline and aztreonam alone and in combination with avibactam against European Gram-negative and Gram-positive clinical isolates. Int J Antimicrob Agents. 2015;45(6):641-646. doi:10.1016/j.ijantimicag.2014.12.033

14. Vena A, Giacobbe DR, Castaldo N, et al. Clinical experience with ceftazidime-avibactam for the treatment of infections due to multidrug-resistant Gram-negative bacteria other than carbapenem-resistant enterobacterales. Antibiotics (Basel, Switzerland). 2020;9(2). doi:10.3390/ antibiotics 9020071

15. Sousa A, Pérez-Rodríguez MT, Soto A, et al. Effectiveness of ceftazidime/avibactam as salvage therapy for treatment of infections due to OXA-48 carbapenemase-producing Enterobacteriaceae. J Antimicrob Chemother. 2018;73(11):3170-3175. doi:10.1093/jac/dky295

16. Rodríguez-Núñez O, Ripa M, Morata L, et al. Evaluation of ceftazidime/avibactam for serious infections due to multidrug-resistant and extensively drug-resistant Pseudomonas aeruginosa. J Glob Antimicrob Resist. 2018;15:136-139. doi:10.1016/j.jgar.2018.07.010

17. Shields RK, Nguyen MH, Chen L, et al. Ceftazidime-avibactam is superior to other treatment regimens against carbapenem-resistant Klebsiella pneumoniae bacteremia. Antimicrob Agents Chemother. 2017;61(8):Aug. doi:10.1128/aac.00883-17

18. van Duin D, Lok JJ, Earley M, et al. Colistin versus ceftazidime-avibactam in the treatment of infections due to carbapenem-resistant Enterobacteriaceae. Clin Infect Dis. 2018;66(2):163-171. doi:10.1093/cid/cix783

19. Jorgensen SCJ, Trinh TD, Zasowski EJ, et al. Real-world experience with ceftazidime-avibactam for multidrug-resistant Gram-negative bacterial infections. Open Forum Infect Dis. 2019;6(12):ofz522. doi:10.1093/ofid/ofz522

20. Tsolaki V, Mantzarlis K, Mpakalis A, et al. Ceftazidime-avibactam to treat life-threatening infections by carbapenem-resistant pathogens in critically Ill mechanically ventilated patients. Antimicrob Agents Chemother. 2020;64(3). doi:10.1128/aac.02320-19

21. Charlson ME, Pompei P, Ales KL, MacKenzie CR. A new method of classifying prognostic comorbidity in longitudinal studies: development and validation. J Chronic Dis. 1987;40(5):373-383. doi:10.1016/0021-9681(87)90171-8

22. Singer M, Deutschman CS, Seymour CW, et al. The Third International Consensus Definitions for Sepsis and Septic Shock (Sepsis-3). JAMA. 2016;315(8):801-810. doi:10.1001/jama.2016.0287

23. Miller JM, Binnicker MJ, Campbell S, et al. A guide to utilization of the microbiology laboratory for diagnosis of infectious diseases: 2018 update by the Infectious Diseases Society of America and the American Society for Microbiology. Clin Infect Dis. 2018;67(6):e1-e94. doi:10.1093/cid/ ciy381

24. Solomkin JS, Mazuski JE, Bradley JS, et al. Diagnosis and management of complicated intra-abdominal infection in adults and children: guidelines by the Surgical Infection Society and the Infectious Diseases Society of America. Clin Infect Dis. 2010;50(2):133-164. doi:10.1086/649554

25. Hooton TM, Bradley SF, Cardenas DD, et al. Diagnosis, prevention, and treatment of catheter-associated urinary tract infection in adults: 2009 International Clinical Practice Guidelines from the Infectious Diseases Society of America. Clin Infect Dis. 2010;50(5):625-663. doi:10.1086/ 650482 
26. Stevens DL, Bisno AL, Chambers HF, et al. Practice guidelines for the diagnosis and management of skin and soft tissue infections: 2014 update by the Infectious Diseases Society of America. Clin Infect Dis. 2014;59(2):e10-52. doi:10.1093/cid/ciu444

27. Tunkel AR, Hasbun R, Bhimraj A, et al. 2017 Infectious Diseases Society of America's Clinical Practice Guidelines for Healthcare-Associated Ventriculitis and Meningitis. Clin Infect Dis. 2017;64(6):e34-e65. doi:10.1093/cid/ciw861

28. Shane AL, Mody RK, Crump JA, et al. 2017 Infectious Diseases Society of America Clinical Practice Guidelines for the Diagnosis and Management of Infectious Diarrhea. Clin Infect Dis. 2017;65(12):e45-e80. doi:10.1093/cid/cix669

29. CLSI. Performance Standards for Antimicrobial Susceptibility Testing. M100 31st ed. Wayne, PA: Clinical and Laboratory Standards Institute; 2021.

30. Alatoom A, Elsayed H, Lawlor K, et al. Comparison of antimicrobial activity between ceftolozane-tazobactam and ceftazidime-avibactam against multidrug-resistant isolates of Escherichia coli, Klebsiella pneumoniae, and Pseudomonas aeruginosa. Int J Infect Dis. 2017;62:39-43. doi:10.1016/ j.ijid.2017.06.007

31. Yang Y, Guo Y, Yin D, et al. In vitro activity of cefepime-zidebactam, ceftazidime-avibactam, and other comparators against clinical isolates of enterobacterales, pseudomonas aeruginosa, and Acinetobacter baumannii: results from China Antimicrobial Surveillance Network (CHINET) in 2018. Antimicrob Agents Chemother. 2020;65(1). doi:10.1128/aac.01726-20

32. Hu YY, Cao JM, Yang Q, et al. Risk factors for carbapenem-resistant Pseudomonas aeruginosa, Zhejiang Province, China. Emerg Infect Dis. 2019;25(10):1861-1867. doi:10.3201/eid2510.181699

33. Tumbarello M, Trecarichi EM, Corona A, et al. Efficacy of ceftazidime-avibactam salvage therapy in patients with infections caused by Klebsiella pneumoniae carbapenemase-producing K. pneumoniae. Clin Infect Dis. 2019;68(3):355-364. doi:10.1093/cid/ciy492

34. Shirley M. Ceftazidime-avibactam: a review in the treatment of serious Gram-negative bacterial infections. Drugs. 2018;78(6):675-692. doi:10.1007/s40265-018-0902-x

35. Montero MM, Domene Ochoa S, López-Causapé C, et al. Time-kill evaluation of antibiotic combinations containing ceftazidime-avibactam against extensively drug-resistant Pseudomonas aeruginosa and their potential role against ceftazidime-avibactam-resistant isolates. Microbiol Spectr. 2021;9(1):e0058521. doi:10.1128/Spectrum.00585-21

36. Pogue JM, Bonomo RA, Kaye KS. Ceftazidime/avibactam, meropenem/vaborbactam, or both? Clinical and formulary considerations. Clin Infect Dis. 2019;68(3):519-524. doi:10.1093/cid/ciy576

37. Hakeam HA, Alsahli H, Albabtain L, Alassaf S, Al Duhailib Z, Althawadi S. Effectiveness of ceftazidime-avibactam versus colistin in treating carbapenem-resistant Enterobacteriaceae bacteremia. Int J Infect Dis. 2021;109:1-7. doi:10.1016/j.ijid.2021.05.079

Infection and Drug Resistance

Dovepress

\section{Publish your work in this journal}

Infection and Drug Resistance is an international, peer-reviewed open-access journal that focuses on the optimal treatment of infection (bacterial, fungal and viral) and the development and institution of preventive strategies to minimize the development and spread of resistance. The journal is specifically concerned with the epidemiology of antibiotic resistance and the mechanisms of resistance development and diffusion in both hospitals and the community. The manuscript management system is completely online and includes a very quick and fair peer-review system, which is all easy to use. Visit http://www.dovepress.com/testimonials.php to read real quotes from published authors.

Submit your manuscript here: https://www.dovepress.com/infection-and-drug-resistance-journal 